\title{
Conhecimentos sobre a doença de Chagas e seus vetores em habitantes de área endêmica do Rio Grande do Sul, Brasil
}

\section{Knowledge about Chagas disease and its vectors of individuals from the endemic area of Rio Grande do Sul, Brazil.}

\author{
Luciane d'Avila Rosenthal' (D), Juliana Nunes Vieira' (D), Marcos Marreiro Villela' (D), \\ Tanise Freitas Bianchi' ${ }^{1}$, Sabrina Jeske ${ }^{1}$ (D) \\ 'Programa de Pós-graduação Stricto Sensu em Parasitologia, Departamento de Microbiologia e Parasitologia, Universidade \\ Federal de Pelotas - Pelotas (RS), Brasil.
}

Como citar: Rosenthal LD, Vieira JN, Villela MM, Bianchi TF, Jeske S. Conhecimentos sobre a doença de Chagas e seus vetores em habitantes de área endêmica do Rio Grande do Sul, Brasil. Cad Saúde Colet, 2020;28(3):345-352. https://doi.org/10.1590/1414-462X202028030426

\section{Resumo}

Introdução: A doença de Chagas (DC) é uma endemia relevante na América Latina, contudo poucas pesquisas são realizadas sobre os conhecimentos que a população possui sobre o tema. Objetivo: Verificar o conhecimento que uma população de origem rural do sul do Rio Grande do Sul possui acerca da DC e seus vetores. Método: O estudo foi realizado com a população atendida em um Hospital-Escola de Pelotas, a partir de questionário semiestruturado, no qual foi verificado se as pessoas conseguiam identificar os triatomíneos, se já encontraram "barbeiros" na residência, quais órgãos a DC acomete, entre outras questões. As associações entre as variáveis foram analisadas pelos testes do qui-quadrado e odds ratio (OR). Resultados: Dos 132 participantes, 58,3\% identificaram os vetores, e as variáveis que se mostraram significativas foram morar ou ter morado no município de Canguçu e possuir galinheiro ou chiqueiro no peridomicílio. Já as variáveis residir ou ter residido em casa de pau a pique/torrão/barro ( $p=0,0038 ; O R=3,18)$ e ser proveniente de Canguçu $(p=0,004 ; O R=3,4)$ foram significativamente associadas ao ato de já ter encontrado vetores na residência. Sobre os órgãos que a DC acomete, somente 25 pessoas reportaram o coração (18,9\%). Conclusão: Esta investigação ressalta a necessidade de campanhas educativas, pois foi realizada em região com importantes índices de DC em estudos recentes.

Palavras-chave: controle; vigilância entomológica; Trypanosoma cruzi; vetores.
Abstract

Background: Chagas disease (CD) is a relevant endemic in Latin America, however, few researches is carried out about the knowledge that the population has on the subject. Objective: Verify the knowledge that the population of rural origin, in the southern region of Rio Grande do Sul, has about the CD and its vectors. Method: The study was carried out with individuals attended at the School Hospital of Pelotas, from a semi-structured questionnaire, through which it was verified if people could identify the vectors; if individuals found "kissing-bugs" in your residence; which organs the CD affects; among other issues. The associations between the variables were analyzed by the $X 2$ and odds ratio (OR) tests. Results: Of the 132 individuals interviewed, 58.3\% identified the significant vectors linked to the variables as to reside or had resided in the municipality of Canguçu and had a chicken coop or pigsty in the peridomicile. The variables residing or having resided in a stick house $(p=0.0038$, $O R=3.18)$ and coming from Canguçu $(p=0.004, O R=3.4)$ were significant to have already found 
vectors in the residence. Regarding the organs that CD affects, only 25 people reported heart issues (18.9\%). Conclusion: This research highlights the need for educational campaigns, being carried out in a region with important $C D$ indexes in recent studies.

Keywords: control; entomological surveillance; Trypanosoma cruzi; vectors.

\section{INTRODUÇÃO}

A doença de Chagas (DC), também intitulada tripanossomíase americana, configura uma endemia de grande relevância na América Latina, a qual acomete, principalmente, populações suscetíveis de zonas rurais que vivem em precárias condições de habitação' ${ }^{1}$. Essa doença é originada de uma infecção causada pelo protozoário flagelado Trypanosoma cruzi (Chagas, 1909), e a via mais importante de transmissão ao homem é a vetorial, por meio das dejeções do triatomíneo infectado (fezes e urina), liberadas durante ou logo após o repasto sanguíneo ${ }^{2,3}$. Outras formas de transmissão são: via oral, transfusional, via vertical/gravidez e transplante de órgãos ${ }^{3}$.

No Brasil, apesar do surgimento eventual de novos casos, foi observada notável diminuição da transmissão da DC a partir das campanhas de controle vetorial, que passaram a ser exercidas de forma regular no país a partir de $1975^{4,5}$. Como a moléstia tende à cronificação e o seu tratamento pode envolver cirurgias cardíacas e digestivas, implante de marcapasso e até transplante cardíaco, o custo econômico da enfermidade é muito elevado. Existe também um elemento importante que é o custo previdenciário da doença em virtude das aposentadorias precoces ${ }^{6}$. A quantidade aproximada de mortes anuais por DC na América Latina é de aproximadamente 14 mil casos $^{7}$, ocorrendo 6 mil só no Brasili ${ }^{8}$. Ademais, há indícios da persistência de transmissão vetorial em algumas regiões, sendo que a permanência do vetor infestando as unidades domiciliares se encontra diretamente associada a fatores econômicos, sociais e ambientais ${ }^{9,10}$.

Alguns estados brasileiros, como Minas Gerais (MG), Goiás (GO), Distrito Federal (DF) e Rio Grande do Sul (RS), apresentam alta frequência de mortes anuais por DC, com elevado número de indivíduos cronicamente infectados ${ }^{11,12}$. O combate à DC no Brasil, desde seu princípio, visou ao controle de vetores no ambiente domiciliar ${ }^{1}$, sendo conduzido em forma de campanha na década de 1950, alcançando toda a área endêmica em meados de 1980, por meio do Programa de Controle da Doença de Chagas (PCDCh), sob coordenação e execução direta do governo federal via Superintendência de Campanhas de Saúde Pública (SUCAM) ${ }^{9}$. PCDCh foi inicialmente dividido em três fases: (a) planejamento e reconhecimento geográfico da área endêmica; (b) fase de ataque aos vetores domiciliados, com expurgos periódicos dos domicílios e seus anexos; (c) vigilância entomológica, para evitar o retorno do principal vetor, até então Triatoma infestans (Klug, 1834) ${ }^{5}$. Durante a fase de vigilância, a participação da comunidade por meio da notificação de insetos suspeitos de serem triatomíneos faz-se fundamental, e projetos educacionais e de esclarecimentos são considerados importantes para manter a população ativa e participativa ${ }^{13}$.

Os triatomíneos que apresentam potencial de colonização do ambiente domiciliar não são encontrados uniformemente distribuídos pelas áreas rurais dos municípios; eles tendem a concentrar-se nas localidades que apresentam habitações favoráveis à sua domiciliação ${ }^{14}$. Logo, a sensibilização e a participação da população são essenciais, sendo que investigações para a compreensão do nível de conhecimento/envolvimento da comunidade são tidas como ferramenta para o desenvolvimento de práticas e ações preventivas ${ }^{15}$. No entanto, são escassos os trabalhos que envolvem o conhecimento e a percepção da população sobre endemias, embora importantes para políticas públicas de prevenção, visto que a falta de conhecimento sobre os vetores, no caso particular da DC, torna-se um óbice diante do controle da enfermidade, como reportado para algumas regiões do país e da América Latina ${ }^{16-19}$.

O Rio Grande do Sul é considerado endêmico para DC, com ênfase para a região sul do estado ${ }^{14}$, incluindo o encontro de insetos vetores em vivendas rurais, mesmo na última década ${ }^{20}$. Com relação ao vetor T. infestans, o estado do Rio Grande do Sul, ao lado da Bahia, constituía um dos poucos locais com focos remanescentes dessa espécie no Brasil, considerada o principal vetor do protozoário nos países do Cone-Sul21. Justificado pelo exposto, este estudo 
objetivou verificar o conhecimento que uma população adulta de origem rural, da região sul do Rio Grande do Sul, possuía acerca da DC e seus vetores.

\section{MÉTODO}

\section{População do estudo}

Foram convidados a participar da pesquisa pacientes internados no Hospital-Escola (HE) da Universidade Federal de Pelotas (UFPel), em Pelotas, Rio Grande do Sul, Brasil, que moravam ou haviam morado na região rural dos municípios da região sul do Rio Grande do Sul. A abordagem foi realizada entre março e agosto de 2016.

O tamanho amostral (132 pacientes) foi estimado pelo número de pacientes internados no HE-UFPel durante o período das entrevistas (aproximadamente 200 pacientes), com nível de confiança de $95 \%$ e margem de erro de $5 \%$, associado à prevalência estimada da DC na região (3\%). Os critérios de inclusão empregados foram: ter realizado a internação durante a pesquisa, ser maior de idade e residir ou ter residido no ambiente rural. As entrevistas eram realizadas nos quartos em que os pacientes estavam internados. Cabe informar que o HospitalEscola atende pacientes oriundos de 22 municípios da região sul do Rio Grande do Sul, o que permite maior representatividade da área de estudo.

A participação na pesquisa foi voluntária, sendo antecedida de explicação dos objetivos aos participantes para posterior assinatura do Termo de Consentimento Livre e Esclarecido (TCLE). O projeto foi avaliado e autorizado pelo Comitê de Ética em Pesquisa da Faculdade de Medicina da UFPel, sob o número 1001545.

\section{Avaliação dos conhecimentos da população}

A identificação dos triatomíneos pelos entrevistados foi avaliada com base em um questionário semiestruturado, acompanhado de mostruário com cinco figuras de artrópodes, em tamanho real, sendo dois triatomíneos adultos, T. infestans e Triatoma rubrovaria (Blanchard, 1843), uma aranha, um carrapato e um hemíptero fitófago (popularmente conhecido na região como "maria-fedida" ou "fede-fede"). Após a apresentação da pesquisa, as seguintes questões foram realizadas:

a) Você reconhece algum destes artrópodes ("bichos")?

b) Se sim, como ele é conhecido (nome do artrópode)?

c) Você já encontrou insetos "barbeiros/chupões" em sua residência?

d) Você conhece a doença de Chagas?

e) Sabe que órgãos (partes do corpo) esta doença pode atingir?

f) Sabe dizer como podemos adquirir a doença (como é transmitida)?

g) Onde aprendeu sobre a doença de Chagas e seus vetores?

h) Sabe se você ou alguém da sua família tem/tinha esta doença?

Considerações: a questão a) era considerada resposta correta se ao menos uma das espécies de "barbeiro" fosse apontada no mostruário; a questão c) foi realizada a todos os participantes, mesmo aqueles que não souberam identificar os vetores, já que os triatomíneos eram identificados pelo entrevistador após a questão b); ainda, a questão c) era relacionada ao município de origem do entrevistado; logo após a pergunta da questão f), foram lidas as seguintes alternativas ao entrevistado ( $\mathrm{i}$ - via vetorial, barbeiro ou chupão; ii - via oral, ingestão de alimentos contaminados; iii - transmissão sanguínea, transfusão de sangue; iv - via vertical, da mãe para o feto; $v$ - por picada de mosquito). Para as demais perguntas, não havia leitura de alternativas, e as respostas eram emitidas de modo espontâneo.

\section{Análise estatística}

As informações coletadas foram digitadas em planilha do programa Microsoft Excel ${ }^{\circledR}$. As associações dos grupos foram realizadas entre as perguntas e as seguintes variáveis, também colhidas na entrevista: idade ( 20 a 59 anos e 60 a 79 anos); grau de instrução (até 4 anos de 
estudo e 5 anos ou mais); renda familiar (até 1 salário mínimo e mais de 1 salário mínimo); só ter morado em casa de alvenaria (sim ou não); residir ou ter residido em casa de pau a pique/ torrão/barro (sim ou não); ser proveniente do município de Canguçu (RS) (sim ou não); possuir galinheiro ou chiqueiro no peridomicílio (sim ou não). A análise estatística foi realizada pelo teste do qui-quadrado, sendo considerados significativos resultados com valor de $p \leq 0,05$. Para valores significativos, também foi procedida a razão de chances para cada variável (Odds ratio, OR). O programa utilizado para análise estatística foi o Minitab ${ }^{\circ}$, versão XVIII. Cumpre informar que a variável ser "proveniente de Canguçu" foi adotada em decorrência da elevada prevalência que a DC apresenta nos moradores provenientes desse município em décadas passadas, até em avaliações mais recentes.

\section{RESULTADOS}

Participaram da pesquisa 132 pessoas pertencentes a 18 municípios da região sul do Rio Grande do Sul que moravam ou haviam morado no meio rural dessa localidade. Desse total, $51,5 \%$ dos pacientes tinham 59 anos ou menos de idade; $78,8 \%$ possuíam até o ensino fundamental completo, dos quais 8,3\% eram analfabetos; e $75 \%$ recebiam até 2 salários mínimos.

Quanto ao reconhecimento dos vetores nos mostruários, 58,3\% (77) apontaram corretamente um ou mais triatomíneos. As variáveis morar ou ter morado no município de Canguçu e possuir galinheiro ou chiqueiro no peridomicílio foram significativamente associadas à correta identificação do inseto (Tabela 1), sendo que T. infestans foi a espécie de triatomíneo mais identificada no mostruário, com 48,5\%, em comparação com 40,2\% que reconheceram T. rubrovaria. Das 77 pessoas que reconheceram os vetores, $49(63,6 \%)$ disseram conhecer o inseto pelo nome de "chupão", 25 (32,5\%) falaram "barbeiro" e 3 (3,9\%) conheciam pelas duas nomenclaturas.

Tabela 1. Associação da identificação dos vetores da doença de Chagas, por pacientes da região sul do Rio Grande do Sul, em relação ao município de origem e presença de galinheiro/chiqueiro no peridomicílio, no período de março a agosto de 2016

\begin{tabular}{|c|c|c|c|c|}
\hline \multirow{2}{*}{ Variáveis } & \multicolumn{2}{|c|}{ Soube identificar os triatomíneos? } & \multirow{2}{*}{ Valor de $p$} & \multirow{2}{*}{$\begin{array}{l}\text { Odds Ratio } \\
\left(\text { IC }_{95 \%}\right)\end{array}$} \\
\hline & Sim (\%) & Não (\%) & & \\
\hline \multicolumn{5}{|c|}{ Município de origem } \\
\hline Canguçu & $28(87,5)$ & $04(12,5)$ & \multirow{2}{*}{0,0005} & \multirow{2}{*}{$7,29(2,38-22,29)$} \\
\hline Outros* & $49(49)$ & $51(51)$ & & \\
\hline \multicolumn{5}{|c|}{ Possuir galinheiro/chiqueiro } \\
\hline Sim & $72(62,6)$ & $43(37,4)$ & \multirow{2}{*}{0,014} & \multirow{2}{*}{$4,02(1,33-12,19)$} \\
\hline Não & $05(29,4)$ & $12(70,6)$ & & \\
\hline
\end{tabular}

* Arroio Grande (11**), Capão do Leão (1), Cerrito (1), Cristal (1), Herval (2), Jaguarão (3), Morro Redondo (1), Pedras Altas (1), Pedro Osório (7), Pelotas (37), Pinheiro Machado (3), Piratini (6), São Lourenço do Sul (11), Santa Vitória do Palmar (4), Santana da Boa Vista (4), Rio Grande (4) e Turuçu (1); ** Número de entrevistados do município.

Quando perguntados "você já encontrou insetos'barbeiros/chupões' em sua residência?", $31,8 \%$ (42) dos investigados responderam que sim. Para essa pergunta, a associação com as variáveis morar ou ter morado em casa de pau a pique/torrão/barro e ser proveniente do município de Canguçu foi estatisticamente significativa (Tabela 2). Já o fato de só ter residido em casa de alvenaria foi tido como fator de proteção, e os moradores encontraram vetores significativamente menos vezes nessas construções (Tabela 2).

Sobre conhecer a DC, 87,1\% (115) dos sujeitos da pesquisa responderam afirmativamente. Contudo, quando inquiridos quanto aos órgãos que a doença pode acometer, somente 32 
Tabela 2. Associação do fato de ter encontrado vetores da doença de Chagas na residência, em relação ao município de origem e ao tipo de moradia, de pacientes da região sul do Rio Grande do Sul, Brasil, no período de março a agosto de 2016

\begin{tabular}{|c|c|c|c|c|}
\hline \multirow{2}{*}{ Variáveis } & \multicolumn{2}{|c|}{$\begin{array}{l}\text { Já encontrou "barbeiros/chupões" na } \\
\text { residência? }\end{array}$} & \multirow{2}{*}{ Valor de $p$} & \multirow{2}{*}{$\begin{array}{l}\text { Odds Ratio } \\
\left.\text { (IC }{ }_{95 \%}\right)\end{array}$} \\
\hline & $\operatorname{Sim}(\%)$ & Não (\%) & & \\
\hline \multicolumn{5}{|c|}{ Morou em casa de pau a pique* } \\
\hline Sim & $20(50)$ & $20(50)$ & \multirow{2}{*}{0,0038} & \multirow{2}{*}{$3,18(1,45-6,96)$} \\
\hline Nunca & $22(23,9)$ & $70(76,1)$ & & \\
\hline \multicolumn{5}{|c|}{ Município de origem } \\
\hline Canguçu & $17(53,1)$ & $15(46,9)$ & \multirow{2}{*}{0,004} & \multirow{2}{*}{$3,40(1,48-7,79)$} \\
\hline Outros & $25(25)$ & $75(75)$ & & \\
\hline \multicolumn{5}{|l|}{ Alvenaria** } \\
\hline Sim & $10(17.5)$ & $47(82,5)$ & \multirow{2}{*}{0,0028} & \multirow{2}{*}{$0,29(0,13-0,65)$} \\
\hline Não & $32(42,7)$ & $43(57,3)$ & & \\
\hline
\end{tabular}

*pau a pique/adobe/torrão/barro. **só residiu em casa de alvenaria.

$(24,2 \%)$ falaram corretamente, dos quais 25 reportaram o acometimento cardíaco (18,9\%), 5 $(3,8 \%)$ mencionaram as alterações cardíacas e digestivas e 2 comentaram apenas os prejuízos ao sistema digestivo (1,5\%), não havendo diferença significativa para a associação das variáveis testadas. Para a questão "sabe dizer como podemos adquirir a doença (como é transmitida)?", para a qual foram lidas as alternativas e o entrevistado poderia apontar mais de uma resposta, $77(58,3 \%)$ reportaram a via vetorial/“barbeiro ou chupão", 46 (34,8\%), a via transfusional, 35 (26,5\%), a via oral/ingestão de alimentos contaminados, 29 (21,9\%), a via vertical, e $13(9,8 \%)$, que a transmissão pode se dar por picada de mosquito.

No que se refere aos locais ou grupos em que os entrevistados aprenderam sobre a parasitose e seus vetores, foi possível destacar a televisão (31,8\%, 42 indivíduos) e o centro familiar (31,1\%, 41 entrevistados) (Figura 1).

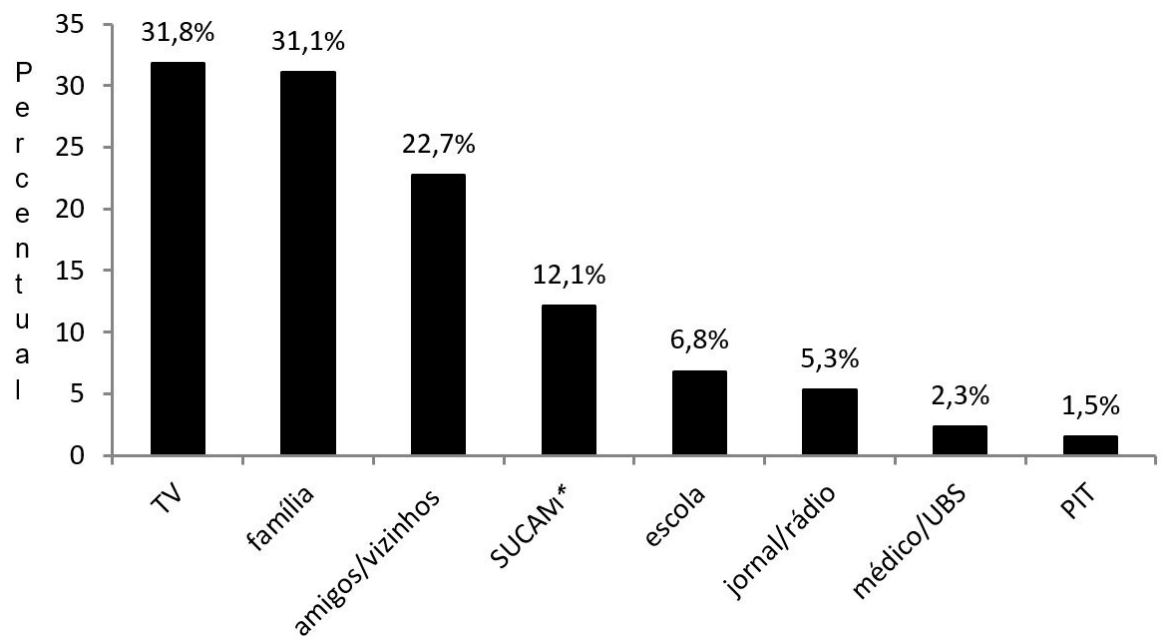

Figura 1. Locais, meios ou grupos nos quais os entrevistados da região sul do Rio Grande do Sul reportaram ter aprendido sobre a doença de Chagas e seus vetores, 2016. *SUCAM -Superintendência de Campanhas de Saúde Pública; UBS - Unidade Básica de Saúde; PIT - Posto de Informação de Triatomíneo. 
Com relação a possuir alguém da família com DC ou ser portador da moléstia, 24 responderam afirmativamente $(18,2 \%)$. Cabe informar que essas pessoas relataram ter encontrado insetos "chupões" em suas residências significativamente mais vezes ( $p=0,02$; $\mathrm{OR}=2,9 ; \mathrm{IC} 95 \%=1,3-6,9)$ do que os participantes que não comentaram ter ou possuir alguém da família com a doença. Nenhum entrevistado falou ser positivo para DC, mas três comentaram que suas mães eram positivas.

\section{DISCUSSÃO}

Dos participantes entrevistados, 58,3\% (77) apontaram corretamente o inseto "chupão", valor inferior aos encontrados por Maeda \& Gurgel-Gonçalves ${ }^{19}$, que, ao estudarem os conhecimentos e práticas de moradores do Distrito Federal (Brasil), em relação à DC e seus vetores, verificaram que $77 \%$ dos entrevistados da região de Águas Claras (área urbana) e $86 \%$ dos de Planaltina (área rural) souberam identificar os vetores. O índice de acerto deste estudo também ficou inferior aos encontrados por Villela e colaboradores ${ }^{16}$, que avaliaram os conhecimentos e práticas que adultos e crianças tinham acerca da DC e triatomíneos em Bambuí (MG). Na ocasião, os autores encontraram $89,1 \%$ de acerto na identificação dos vetores para adultos e $66,7 \%$ para escolares. Nesse ponto, é preciso frisar que a cidade de Bambuí é tida como municipalidade-modelo para o controle da DC, tendo, inclusive, um posto avançado de pesquisa há mais de 70 anos sediado no município, para abordar a epidemiologia e o controle da enfermidade ${ }^{16}$ e, por tal motivo, provavelmente as pessoas estão mais sensibilizadas para identificar os vetores da moléstia.

Respaldando a maior identificação dos "barbeiros" realizados por moradores de Canguçu, Baruffa e Alcântara ${ }^{14}$ encontraram nos municípios de Canguçu e Piratini (cidades vizinhas) os maiores focos de triatomíneos nos domicílios, além de casos agudos da DC, especialmente pelo tipo de moradia existente na época. A prevalência elevada de infecção por T. cruzi em pacientes provenientes de Canguçu ainda é encontrada em estudos atuais ${ }^{22}$. Assim, o elevado índice de insetos vetores capturados no passado, provavelmente, seja o que faz com que população desse município esteja sensibilizada para a identificação dos "barbeiros".

No que tange à associação entre as variáveis conhecer triatomíneos e possuir galinheiro na unidade domiciliar, a presença de animais de sangue quente no peridomicílio pode atrair insetos hematófagos para o entorno da residência, e, por esse motivo, a população com essas construções no peridomicílio apresenta maior probabilidade de encontrar vetores nesse ambiente. Estudos mostram que o sangue das aves é repetidamente encontrado no tubo digestório dos triatomíneos capturados próximos a construções humanas ${ }^{23}$. Com isso, há necessidade de informar às pessoas sobre a importância de manter o peridomicílio organizado e inspecionado quanto à presença de triatomíneos, visto que são observados focos de "chupões" em casas de boa construção, mas localizadas próximas de anexos precários e de má qualidade. Além disso, esta atenção ao peridomicílio deve ser reforçada, pois a resposta que as medidas de controle possuem nas áreas extradomiciliares é diminuída, já que os inseticidas se deterioram mais rapidamente ${ }^{16,24,25}$.

Foi percebido que a maior parte dos participantes conhecia os triatomíneos vetores como "chupão"; assim, seria mais adequado o uso dessa nomenclatura no decorrer das campanhas contra esses vetores no sul do Rio Grande do Sul. Além disso, durante as ações educativas, o vocabulário utilizado deve ser coerente com a mensagem e o público-alvo, utilizando palavras com definições simples e familiares ${ }^{26}$. Uma linguagem simples, que comunique uma mensagem culturalmente adequada, pode diminuir as barreiras da comunicação, tornando-a mais eficiente ${ }^{27,28}$.

Em relação à associação de residir/ter residido em casas de pau a pique, ser natural de Canguçu, com o maior encontro de barbeiros chupões na residência, tal achado está de acordo com o diagnosticado por Rosenthal e colaboradores ${ }^{22}$, uma vez que esses autores verificaram que essas variáveis elevavam a possibilidade de ser positivo para $T$. cruzi em 8,86 e 6,57 vezes, respectivamente. Já o fato de só ter habitado casa de alvenaria, ainda que no meio rural, foi encontrado como fator de proteção, sendo que as frestas e as rachaduras presentes em casas de pau a pique/torrão/barro constituem o principal esconderijo para os vetores ${ }^{29}$. 
Outro desfecho importante refere-se ao menor conhecimento da população avaliada em relação à doença propriamente dita, quando comparado a outros estudos. Maeda e GurgelGonçalves ${ }^{19}$ verificaram que $34 \%$ da população de Águas Claras (Distrito Federal) e $26,41 \%$ da população de Planaltina (Goiás) sabiam quais órgãos eram afetados pela doença, em comparação aos $24,2 \%$ dos entrevistados desta pesquisa. Por sua vez, Dias et al. verificaram que $72,96 \%$ dos entrevistados em Diamantina (MG) reportaram o coração como principal órgão acometido pela DC. Contudo, é preciso considerar que existiam alternativas no questionário, o que pode ter induzido ou facilitado a lembrança de algumas respostas, diferentemente do que ocorreu na presente pesquisa, já que as declarações eram espontâneas para essa questão.

A sustentabilidade das ações de prevenção e controle da DC passa, obrigatoriamente, pela informação e participação da população. Porém, o grande problema é que no Brasil, via de regra, o Ministério da Saúde e as Secretarias Estaduais de Saúde não têm equipes bem estruturadas e atuantes no ramo educativo. Também não há ações estruturadas na educação formal que priorizem as atividades pertinentes a esses agravos ${ }^{16,30}$. $O$ aprendizado sobre a doença a partir de informações fornecidas por médicos, UBS (Unidade Básica de Saúde) e os PITs (Posto de Informação de Triatomíneos), os quais deveriam servir de pilares para divulgação da enfermidade, foi o mesmo reportado pelos participantes. Merece menção que uma breve divulgação dos PITs foi promovida pelos entrevistadores aos sujeitos pesquisados.

Este estudo indica a percepção e compreensão de adultos associados à DC e seus vetores, o que preenche uma lacuna na literatura relativamente escassa sobre o assunto, em uma região endêmica. Assim, é possível concluir que, embora a área avaliada seja de importância epidemiológica para a DC, o conhecimento que a população apresenta sobre essa endemia e seus vetores pode ser considerado escasso, especialmente quando comparado com outras investigações realizadas no país, com metodologia semelhante. Logo, intervenções dessa natureza mostram-se relevantes para fomentar e manter o controle da endemia, uma vez que pesquisas com esse foco, normalmente, apresentam desdobramento pedagógico, fazendo com que a população venha a relembrar aspectos e conceitos sobre a doença estudada e seus vetores.

\section{REFERÊNCIAS}

1. Dias JVL, Queiroz DRM, Diotaiuti L, Pires HHR. Conhecimentos sobre triatomíneos e sobre a doença de Chagas em localidades com diferentes níveis de infestação vetorial. Cien Saude Colet. 2016;21(7):2293304. http://dx.doi.org/10.1590/1413-81232015217.07792015. PMid:27383362.

2. Silveira AC. Situação do controle da transmissão vetorial da doença de Chagas nas Américas. Cad Saude Publica. 2000;16(2 Suppl):35-42. http://dx.doi.org/10.1590/S0102-311X2000000800004. PMid:11119318.

3. Luquetti AO, Tavares SBN, Siriano LR, Oliveira RA, Campos DE, Morais CA, et al. Congenital transmission of Trypanosoma cruzi in Central Brazil. A study of 1,211 individuals born to infected mothers. Mem Inst Oswaldo Cruz. 2015;110(3):369-76. http://dx.doi.org/10.1590/0074-02760140410. PMid:25993506.

4. Coura JR, Dias JCP. Epidemiology, control and surveillance of Chagas disease - 100 years after its Discovery. Mem Inst Oswaldo Cruz. 2009;104(1 Suppl):31-40. http://dx.doi.org/10.1590/S0074-02762009000900006. PMid:19753455.

5. Silveira AC, Dias JCP. O controle da transmissão vetorial. Rev Soc Bras Med Trop. 2011;44(2 Suppl):52-63. http://dx.doi.org/10.1590/S0037-86822011000800009. PMid:21584358.

6. Lee BY, Bacon KM, Bottazzi ME, Hotez PJ. Global economic burden of Chagas disease: a computational simulation model. Lancet Infect Dis. 2013;13(4):342-8. http://dx.doi.org/10.1016/S1473-3099(13)70002-1. PMid:23395248.

7. Schmunis GA, Yadon ZE. Chagas disease: A Latin American health problem becoming a world health problem. Acta Trop. 2010;115(1-2):14-21. http://dx.doi.org/10.1016/j.actatropica.2009.11.003. PMid:19932071.

8. Martins Melo FR, Ramos Jr AN, Alencar CH, Heukelbach J. Mortality due to Chagas disease in Brazil from 1979 to 2009: trends and regional differences. J Infect Dev Ctries. 2012;6(11):817-24. http://dx.doi. org/10.3855/jidc.2459. PMid:23277508.

9. Silveira AC, Pimenta F Jr. A inserção institucional do controle da doença de Chagas. Rev Soc Bras Med Trop. 2011;44(2 Suppl):19-24. http://dx.doi.org/10.1590/S0037-86822011000800004. PMid:21584353. 
10. Abad-Franch F, Diotaiuti L, Gurgel-Gonçalves R, Gürtler RE. Certifying the interruption of Chagas disease transmission by native vectors: cui bono? Mem Inst Oswaldo Cruz. 2013;108(2):251-4. http://dx.doi. org/10.1590/0074-0276108022013022. PMid:23579810.

11. da Nóbrega AA, de Araújo WN, Vasconcelos AMN. due to Chagas disease in Brazil according to a specific cause. Am J Trop Med Hyg. 2014;91(3):528-33. http://dx.doi.org/10.4269/ajtmh.13-0574.

12. Moraes CA. Mortalidade por doença de Chagas no estado de Goiás, Brasil no período de 2006 a 2011 [dissertação]. Goiânia: Programa de pós-graduação em Ciências da Saúde. Universidade Federal de Goiás; 2017.

13. Villela MM, Souza JMB, Melo VP, Dias JCP. Avaliação do Programa de Controle da Doença de Chagas em relação à presença de Panstrongylus megistus na região centro-oeste do Estado de Minas Gerais, Brasil. Cad Saude Publica. 2009a;25(4):907-17. http://dx.doi.org/10.1590/S0102-311X2009000400022. PMid:19347217.

14. Baruffa G, Alcantara A Fo. Inquérito sorológico e entomológico da infecção pelo T. cruzi na região Sul do Rio Grande do Sul, Brasil. Ann Soc Belg Med Trop. 1985;65(1 Suppl):171-9. PMid:3931582.

15. Crocco L, Rodríguez C, Catalá S, Nattero J. Enfermedad de Chagas en Argentina: herramientas para que los escolares vigilen y determinen la presencia de factores de riesgo en sus viviendas. Cad Saude Publica. 2005;21(2):646-51. http://dx.doi.org/10.1590/S0102-311X2005000200034. PMid:15905931.

16. Villela MM, Pimenta DN, Lamounier PA, Dias JCP. Avaliação de conhecimentos e práticas que adultos e crianças têm acerca da doença de Chagas e seus vetores em região endêmica de Minas Gerais, Brasil. Cad Saude Publica. 2009b;25(8):1701-10. http://dx.doi.org/10.1590/S0102-311X2009000800006. PMid:19649411.

17. Trivedi M, Sanghavi D. Knowledge deficits regarding Chagas disease may place Mexico's blood supply at risk. Transfus Apher Sci. 2010;43(2):193-6. http://dx.doi.org/10.1016/j.transci.2010.07.008. PMid:20688572.

18. Siqueira-Batista R, Gomes AP, Rôças G, Cotta RMM, Rubião ECN, Pissinatti A. Moléstia de Chagas e ecologia profunda: a"luta antivetorial" em questão. Cien Saude Colet. 2011;16(2):677-87. http://dx.doi.org/10.1590/ S1413-81232011000200032. PMid:21340344.

19. Maeda MH, Gurgel-Gonçalves R. Conhecimentos e práticas de moradores do Distrito Federal, Brasil, em relação à doença de chagas e seus vetores. Rev Patol Trop. 2012;41(1):15-26. http://dx.doi.org/10.5216/ rpt.v41i1.17749.

20. Priotto MCM, Santos CV, Mello F, Ferraz ML, Villela MM. Aspectos da vigilância entomológica da doença de Chagas no sul do Rio Grande do Sul, Brasil. Rev Patol Trop. 2014;43(2):228-38.

21. Bedin C, Mello F, Wilhelm TS, Torres MA, Estima C, Ferreira CF, Sehn L. Vigilância Ambiental: Doença de Chagas no Rio Grande do Sul. Bol Epidemiológico. 2009;11(3):1-8.

22. Rosenthal LA, Petrarca CR, Mesenburg MA, Villela MM. Trypanosoma cruzi seroprevalence and associated risk factors in cancer patients from Southern Brazil. Rev Soc Bras Med Trop. 2016;49(6):768-71. http:// dx.doi.org/10.1590/0037-8682-0202-2016. PMid:28001226.

23. Villela MM, Rodrigues VLC, Casanova CC, Dias JCP. Análise da fonte alimentar de Panstrongylus megistus (Hemiptera, Reduviidae, Triatominae) e sua atual importância como vetor do Trypanosoma cruzi, no Estado de Minas Gerais. Rev Soc Bras Med Trop. 2010;43(2):125-8. http://dx.doi.org/10.1590/S003786822010000200004. PMid:20464139.

24. Diotaiuti L, Pinto CT. Suscetibilidade biológica do Triatoma sordida e Triatoma infestans a deltametrina e lambdacyhalotrina em condições de campo. Rev Soc Bras Med Trop. 1991;24(3):151-5. http://dx.doi. org/10.1590/S0037-86821991000300005. PMid:1842841.

25. Jörg ME. La modificación del biotopo perihabitacional en la profilaxis de la enfermedad de Chagas. Rev Soc Bras Med Trop. 1989;22(2):91-5. http://dx.doi.org/10.1590/S0037-86821989000200005. PMid:2517852.

26. Vanoye F. Usos da linguagem: problemas e técnicas na produção oral e escrita. $11^{\text {a }}$ ed. São Paulo: Martins Fontes; 1998. 327 p.

27. Stefanelli MC. Comunicação com paciente: teoria e ensino. 2a ed. São Paulo: Robe; 1993. 200 p.

28. Moreira MDF, Lima Da Nóbrega, MM, Tabosa da Silva MI. (2003). Comunicação escrita: contribuição para a elaboração de material educativo em saúde. Rev bras enferm. 2009;56(2):184-188. http://dx.doi. org/10.1590/S0034-71672003000200015.

29. Rocha e Silva EO, Rodrigues VLCC, Silva RA, Wanderley DMV. Programa de Controle da Doença de Chagas no Estado de São Paulo, Brasil: o controle e a vigilância da transmissão vetorial. Rev Soc Bras Med Trop. 2011;44(2 Suppl):74-84. http://dx.doi.org/10.1590/S0037-86822011000800012.

30. Dias JCP. Evolution of chagas disease screening programs and control programs: historical perspective. Glob Heart. 2015;10(3):193-202. http://dx.doi.org/10.1016/j.gheart.2015.06.003. PMid:26407516. 